PROCEEDINGS OF THE

AMERICAN MATHEMATICAL SOCIETY

Volume 127, Number 8, Pages 2353-2366

S 0002-9939(99)04826-1

Article electronically published on April 8, 1999

\title{
EQUIVALENCE RELATIONS AND DISTANCES BETWEEN HILBERT FRAMES
}

\author{
RADU BALAN
}

(Communicated by David R. Larson)

\begin{abstract}
We study some equivalency relations between Hilbert frames and closed subspaces of $l^{2}(\mathbf{I})$. We define also a distance between frames and we establish the geometric meaning of this metric. Finally we find the closest and respectively the nearest tight frame to a given frame.
\end{abstract}

\section{INTRODUCTION}

Suppose $H$ is an infinite dimensional separable Hilbert space. A theorem due to Paley-Wiener [PaWi34] states the following: let $\left\{e_{i}\right\}_{i \in \mathbf{N}}$ be an orthonormal basis of $H$ and let $\left\{f_{i}\right\}_{i \in \mathbf{N}}$ be a family of vectors in $H$. If there exists a constant $\lambda \in[0,1)$ such that

$$
\left\|\sum_{i=1}^{n} c_{i}\left(e_{i}-f_{i}\right)\right\| \leq \lambda\left\|\sum_{i=1}^{n} c_{i} e_{i}\right\|=\lambda\left(\sum_{i=1}^{n}\left|c_{i}\right|^{2}\right)^{1 / 2}
$$

for all $n, c_{1}, c_{2}, \ldots, c_{n}$, then $\left\{f_{i}\right\}_{i \in \mathbf{N}}$ is a Riesz basis in $H$ and a frame with bounds $(1-\lambda)^{2},(1+\lambda)^{2}$. An extension of this theorem was given by Christensen in [Chr95] to Hilbert frames and by Christensen and Heil in [ChHe96] to Banach frames.

Duffin and Eachus ([DuSc52]) proposed a converse of the above result by proving that every Riesz basis, after a proper scaling, is close to an orthonormal basis in the sense of (1.1). We are going to extend this result to Hilbert frames and prove some results about quadratic closeness and distance between two frames.

Let $\mathbf{I}$ be a countable index set. A family of vectors $\mathcal{F}=\left\{f_{i}\right\}_{i \in \mathbf{I}}$ in $H$ is called a (Hilbert) frame if there exist two real numbers $0<A \leq B<\infty$ such that for any $x \in H$ we have:

$$
A\|x\|^{2} \leq \sum_{i \in \mathbf{I}}\left|\left\langle x, f_{i}\right\rangle\right|^{2} \leq B\|x\|^{2} .
$$

If $A=B$ we call the frame tight. The largest constant $A$ and respectively the smallest constant $B$ that satisfy (1.2) are called the (optimal) frame bounds.

To a frame $\mathcal{F}$ we associate several objects. Consider the operator

$$
T: H \rightarrow l^{2}(\mathbf{I}), T(x)=\left(\left\langle x, f_{i}\right\rangle\right)_{i \in \mathbf{I}},
$$

Received by the editors October 31, 1997.

1991 Mathematics Subject Classification. Primary 42C99, 46 C99.

Key words and phrases. Closeness bound, nearness, quadratic distance between frames.

The author is grateful to Ingrid Daubechies for the many hours of working together and for the continuous support and encouragement. He also thanks David Larson for a copy of his paper. 
called the analysis operator associated to $\mathcal{F}$ (see [Ron96] for terminology). From (1.2) we get that it is a bounded operator with norm $\|T\|=\sqrt{B}$ and its range is closed. The adjoint of $T$ is given by

$$
T^{*}: l^{2}(\mathbf{I}) \rightarrow H, T^{*} c=\sum_{i \in \mathbf{I}} c_{i} f_{i}
$$

and is called the synthesis operator. With these two operators we construct the frame operator by

$$
S: H \rightarrow H, S=T^{*} T \text { or } S(x)=\sum_{i \in \mathbf{I}}\left\langle x, f_{i}\right\rangle f_{i} .
$$

The condition (1.2) can then be read as $A \cdot 1 \leq S \leq B \cdot 1$ and therefore the frame bounds are $B=\|S\|, A=\left\|S^{-1}\right\|^{-1}$ (for details, we refer the reader to [DuSc52]).

To every frame $\mathcal{F}$ one can associate two special frames: one is called the (standard) dual frame and the other (less frequently used) is called the associated tight frame (see relation (2.12) in [AAG93]). The (standard) dual frame is defined by

$$
\tilde{\mathcal{F}}=\left\{\tilde{f}_{i}\right\}_{i \in \mathbf{I}}, \tilde{f}_{i}=S^{-1} f_{i}
$$

and has a lot of useful properties. A few of them are the following:

(1) $\tilde{\mathcal{F}}$ is a frame with frame bounds $\frac{1}{B}, \frac{1}{A}$.

(2) If $\tilde{T}$ is the analysis operator associated to $\tilde{\mathcal{F}}$, then $\tilde{T}=T S^{-1}$ and the following resolutions of identity (or reconstruction formulae) hold:

$$
1=\tilde{T}^{*} T=T^{*} \tilde{T} \text { or } x=\sum_{i \in \mathbf{I}}\left\langle x, f_{i}\right\rangle \tilde{f}_{i}=\sum_{i \in \mathbf{I}}\left\langle x, \tilde{f}_{i}\right\rangle f_{i} .
$$

(3) In $l^{2}(\mathbf{I}), T$ and $\tilde{T}$ have the same range $(E=\operatorname{Ran} T=\operatorname{Ran} \tilde{T})$ and $P=$ $T \tilde{T}^{*}=\tilde{T} T^{*}$ is the orthogonal projector onto $E$.

(4) For any $c \in l^{2}(\mathbf{I})$ we can consider the set of sequences $d \in l^{2}(\mathbf{I})$ with the same image as $c$, i.e., $T^{*} c=T^{*} d$; the minimum $l^{2}$-norm in this set is achieved by the sequence $c^{*}=P c \in E$.

The associated tight frame is defined by

$$
\mathcal{F}^{\#}=\left\{f_{i}^{\#}\right\}_{i \in \mathbf{I}}, f_{i}^{\#}=S^{-1 / 2} f_{i} .
$$

A few properties of the associated tight frame that can be simply checked are the following:

(1) The associated tight frame is a tight frame with frame bound 1.

(2) If $T^{\#}$ is the analysis operator associated to $\mathcal{F}^{\#}$, then $T^{\#}=T S^{-1 / 2}$; its range coincides with $E=\operatorname{Ran} T$, and the orthogonal projector onto $E, P$, is also equal to $T^{\#}\left(T^{\#}\right)^{*}$.

We shall come back to this associated tight frame in section 3 .

So far, we have just listed properties of one frame and some derived frames. In this paper we shall discuss mainly the relations between two frames. Let $\mathcal{F}=$ $\left\{f_{i}\right\}_{i \in \mathbf{I}}$ and $\mathcal{G}=\left\{g_{i}\right\}_{i \in \mathbf{I}}$ be two frames in $H$. We define the following notions:

- If $Q$ is an invertible bounded operator $Q: H \rightarrow H$ and if $g_{i}=Q f_{i}$, then we say that $\mathcal{F}$ and $\mathcal{G}$ are $Q$-equivalent.

- We say they are unitarily equivalent if they are $Q$-equivalent for a unitary operator $Q$.

- If $Q$ is a bounded operator $Q: H \rightarrow H$ (not necessarily invertible) and $g_{i}=Q f_{i}$, then we say $\mathcal{F}$ is $Q$-partial equivalent with $\mathcal{G}$. 
- We say $\mathcal{F}$ is partial isometric equivalent with $\mathcal{G}$ if there exists a partial isometry $J: H \rightarrow H$ such that $g_{i}=J f_{i}$ (then $J$ should satisfy $J J^{*}=1$ since $g_{i} \in \operatorname{Ran} J$ and $\mathcal{G}$ is a complete set in $\left.H\right)$.

The last two relations ( $Q$-partial equivalent and partial isometric equivalent) are not equivalency relations, because they are not symmetric.

We say that a frame $\mathcal{G}=\left\{g_{i}\right\}_{i \in \mathbf{I}}$ is (quadratically) close to a frame $\mathcal{F}=\left\{f_{i}\right\}_{i \in \mathbf{I}}$ if there exists a positive number $\lambda \geq 0$ such that

$$
\left\|\sum_{i \in \mathbf{I}} c_{i}\left(g_{i}-f_{i}\right)\right\| \leq \lambda\left\|\sum_{i \in \mathbf{I}} c_{i} f_{i}\right\|
$$

for any $c=\left(c_{i}\right)_{i \in \mathbf{I}} \in l^{2}(\mathbf{I})$ (see [You80]). The infimum of such $\lambda$ 's for which (1.5) holds for any $c \in l^{2}(\mathbf{I})$ will be called the closeness bound of the frame $\mathcal{G}$ to the frame $\mathcal{F}$ and denoted by $c(\mathcal{G}, \mathcal{F})$.

The closeness relation is not an equivalency relation (it is transitive, but not reflexive, in general). However, if $\mathcal{G}$ is quadratically close to $\mathcal{F}$ with a closeness bound less than 1 , then $\mathcal{F}$ is also quadratically close to $\mathcal{G}$ but the closeness bound is different, in general. Indeed, from (1.5) it follows that

$$
\left\|\sum_{i \in \mathbf{I}} c_{i}\left(g_{i}-f_{i}\right)\right\| \leq \frac{\lambda}{1-\lambda}\left\|\sum_{i \in \mathbf{I}} c_{i} g_{i}\right\|
$$

The closeness bound can be related to a relative operator bound used in perturbation theory (see [Kato76]). More specifically, if $T^{g}, T^{f}$ denote the analysis operators associated, respectively, to the frames $\mathcal{G}$ and $\mathcal{F}$, then $c(\mathcal{G}, \mathcal{F})$ is the $\left(T^{f}\right)^{*}$ bound of $\left(T^{g}\right)^{*}-\left(T^{f}\right)^{*}$ (in the terminology of Kato).

The next step is to correct the nonreflexivity of the closeness relation. We say that two frames $\mathcal{F}=\left\{f_{i}\right\}_{i \in \mathbf{I}}$ and $\mathcal{G}=\left\{g_{i}\right\}_{i \in \mathbf{I}}$ are near if $\mathcal{F}$ is close to $\mathcal{G}$ and $\mathcal{G}$ is close to $\mathcal{F}$. It is fairly easy to check that this is an equivalency relation. In this case we define the predistance between $\mathcal{F}$ and $\mathcal{G}$, denoted $d^{0}(\mathcal{F}, \mathcal{G})$ as the maximum between the two closeness bounds:

$$
d^{0}(\mathcal{F}, \mathcal{G})=\max (c(\mathcal{F}, \mathcal{G}), c(\mathcal{G}, \mathcal{F})) .
$$

It is easy to prove that $d^{0}$ is positive and symmetric, but does not satisfy the triangle inequality. This inconvenience can be removed if we define the (quadratic) distance between $\mathcal{F}$ and $\mathcal{G}$ by

$$
d(\mathcal{F}, \mathcal{G})=\log \left(d^{0}(\mathcal{F}, \mathcal{G})+1\right) .
$$

Then, as we shall see later (Theorem 2.7), this is a veritable distance (a metric) on the set of frames which are near to one another.

Since the nearness relation is an equivalency relation, we can partition the set of all frames on $H$, denoted $\mathcal{F}(H)$, into disjoint equivalent classes, indexed by an index set $\mathbf{A}$ :

$$
\mathcal{F}(H)=\bigcup_{\alpha \in A} \mathcal{E}_{\alpha}
$$

with the following properties:

$$
\begin{gathered}
\mathcal{E}_{\alpha} \cap \mathcal{E}_{\beta}=\emptyset, \text { for } \alpha \neq \beta, \\
\forall \mathcal{F}, \mathcal{G} \in \mathcal{E}_{\alpha}, d(\mathcal{F}, \mathcal{G})<\infty \text { and } \forall \mathcal{F} \in \mathcal{E}_{\alpha}, \mathcal{G} \in \mathcal{E}_{\beta} \text { with } \alpha \neq \beta, d(\mathcal{F}, \mathcal{G})=\infty
\end{gathered}
$$


Let $\pi$ denote the index projection $\pi: \mathcal{F}(H) \rightarrow \mathbf{A}$ with $\mathcal{F} \rightarrow \pi(\mathcal{F})=\alpha$ if $\mathcal{F} \in \mathcal{E}_{\alpha}$. We shall prove that the partition (1.8) corresponds to the nondisjoint partition of $l^{2}(\mathbf{I})$ into closed infinite dimensional subspaces. Moreover, the two equivalency relations introduced before are identical (i.e., two frames are near if and only if they are $Q$-equivalent) as we shall prove later.

For a frame $\mathcal{G}$ we denote by $\mathcal{T}^{1}$ the set of tight frames which are quadratically close to $\mathcal{G}$ and by $\mathcal{T}^{2}$ the set of tight frames such that $\mathcal{G}$ is close to them:

$$
\begin{gathered}
\mathcal{T}^{1}=\left\{\mathcal{F}=\left\{f_{i}\right\}_{i \in \mathbf{I}} \mid \mathcal{F} \text { is a tight frame and } c(\mathcal{G}, \mathcal{F})<+\infty\right\}, \\
\mathcal{T}^{2}=\left\{\mathcal{F}=\left\{f_{i}\right\}_{i \in \mathbf{I}} \mid \mathcal{F} \text { is a tight frame and } c(\mathcal{F}, \mathcal{G})<+\infty\right\} .
\end{gathered}
$$

Let $d^{1}: \mathcal{T}^{1} \rightarrow \mathbf{R}_{+}, d^{2}: \mathcal{T}^{2} \rightarrow \mathbf{R}_{+}$denote the map from each $\mathcal{F}$ to the associated closeness bound, i.e., $d^{1}(\mathcal{F})=c(\mathcal{G}, \mathcal{F})$ and $d^{2}(\mathcal{F})=c(\mathcal{F}, \mathcal{G})$. If $\mathcal{G}$ is a tight frame itself, then $\mathcal{G} \in \mathcal{T}^{1} \cap \mathcal{T}^{2}$ and $\min d^{1}=\min d^{2}=0$.

Consider now the intersection between these two sets:

$$
\mathcal{T}=\mathcal{T}^{1} \cap \mathcal{T}^{2}=\left\{\mathcal{F}=\left\{f_{i}\right\}_{i \in \mathbf{I}} \mid \mathcal{F} \text { is a tight frame and } d(\mathcal{F}, \mathcal{G})<+\infty\right\} \subset \mathcal{E}_{\pi(\mathcal{G})} .
$$

In section 3 we will be looking for the minima of the functions $d^{1}, d^{2}$ and $\left.d\right|_{\mathcal{T}}$.

\section{Geometry of Hilbert frames}

In this section we are mainly concerned with the relations introduced previously. We shall prove that $Q$-equivalence is the same as nearness (in other words, two frames are $Q$-equivalent if and only if they are near). The following lemmas are fundamental for all constructions and results in this paper.

Lemma 2.1. Consider $\mathcal{F}_{1}=\left\{f_{i}^{1}\right\}_{i \in \mathbf{I}}$ and $\mathcal{F}_{2}=\left\{f_{i}^{2}\right\}_{i \in \mathbf{I}}$ two tight frames in $H$ with frame bounds 1. Denote by $T_{1}$ and $T_{2}$ respectively their analysis operators. Then:

1) Ran $T_{2} \subset R a n T_{1}$ if and only if $\mathcal{F}_{1}$ and $\mathcal{F}_{2}$ are partial isometric equivalent; moreover, if $J$ is the corresponding partial isometry, then $K$ er $J \simeq R a n T_{1} / \operatorname{Ran} T_{2}$; more specifically $\operatorname{Ker} J=T_{1}^{*}\left(\operatorname{Ran} T_{1} \cap\left(\operatorname{Ran} T_{2}\right)^{\perp}\right)$;

2) Ran $T_{1}=\operatorname{Ran} T_{2}$ if and only if $\mathcal{F}_{1}$ and $\mathcal{F}_{2}$ are unitarily equivalent.

Proof. 1. Suppose $\mathcal{F}_{1}$ and $\mathcal{F}_{2}$ are partial isometric equivalent. Then $f_{i}^{2}=J f_{i}^{2}$ and $T_{2}=T_{1} J^{*}$ for some partial isometry $J$. Obviously, $\operatorname{Ran} T_{2} \subset \operatorname{Ran} T_{1}$. Now, recall that $T_{1}$ and $T_{2}$ are isometries from $H$ onto their ranges (since $\mathcal{F}_{1}$ and $\mathcal{F}_{2}$ are tight frames with bound 1). Therefore they preserve the scalar product and linear independence. Thus,

$$
\operatorname{Ran} T_{1}=T_{1}\left(\operatorname{Ran} J^{*} \oplus \operatorname{Ker} J\right)=T_{1} J^{*}(H) \oplus T_{1}(\text { Ker } J)=\operatorname{Ran} T_{2} \oplus T_{1}(\text { Ker } J)
$$

and $T_{1}(\operatorname{Ker} J)$ is the orthogonal complement of $\operatorname{Ran} T_{2}$ into $\operatorname{Ran} T_{1}$. On the other hand, $\left.T_{1}^{*}\right|_{\operatorname{Ran} T_{1}}$ is the inverse of $T_{1}: H \rightarrow \operatorname{Ran} T_{1}$; thus,

$$
\operatorname{Ker} J=T_{1}^{*}\left(\operatorname{Ran} T_{1} \cap\left(\operatorname{Ran} T_{2}\right)^{\perp}\right)
$$

fixing canonically the isometric isomorphism $\operatorname{Ker} J \simeq \operatorname{Ran} T_{1} / \operatorname{Ran} T_{2}$.

Conversely, suppose $\operatorname{Ran} T_{2} \subset \operatorname{Ran} T_{1}$. Then, the two projectors are $P_{1}=T_{1} T_{1}^{*}$ onto $\operatorname{Ran} T_{1}$ and $P_{2}=T_{2} T_{2}^{*}$ onto $\operatorname{Ran} T_{2}$ and we have $P_{1} T_{2}=T_{2}$. Now, consider $J: H \rightarrow H, J=T_{2}^{*} T_{1}$ which acts in the following way:

$$
J(x)=\sum_{i \in \mathbf{I}}\left\langle x, f_{i}^{1}\right\rangle f_{i}^{2} .
$$


We have

$$
J J^{*}=T_{2}^{*} T_{1} T_{1}^{*} T_{2}=T_{2}^{*} P_{1} T_{2}=T_{2}^{*} T_{2}=1 .
$$

We want to prove now that $f_{j}^{2}=J f_{j}^{1}$ for all $j$. We have, for fixed $j$,

$$
J f_{j}^{1}-f_{j}^{2}=\sum_{i \in \mathbf{I}}\left(\left\langle f_{j}^{1}, f_{i}^{1}\right\rangle-\left\langle f_{j}^{2}, f_{i}^{2}\right\rangle\right) f_{i}^{2}=T_{2}^{*} c
$$

where $c=\left\{c_{i}\right\}_{i \in \mathbf{I}}, c_{i}=\left\langle f_{j}^{1}, f_{i}^{1}\right\rangle-\left\langle f_{j}^{2}, f_{i}^{2}\right\rangle$. On the other hand,

$$
0=f_{j}^{1}-\sum_{i \in \mathbf{I}}\left\langle f_{j}^{1}, f_{i}^{1}\right\rangle f_{i}^{1}=\sum_{i \in \mathbf{I}}\left(\delta_{i j}-\left\langle f_{j}^{1}, f_{i}^{1}\right\rangle\right) f_{i}^{1}=T_{1}^{*} a^{j}
$$

where $a^{j}=\left\{a_{i}^{j}\right\}_{i \in \mathbf{I}}, a_{i}^{j}=\delta_{i j}-\left\langle f_{j}^{1}, f_{i}^{1}\right\rangle$ and $\delta_{i j}$ is the Kronecker symbol. Similarly, $0=T_{2}^{*} b^{j}$ with $b^{j}=\left\{b_{i}^{j}\right\}_{i \in \mathbf{I}}, b_{i}^{j}=\delta_{i j}-\left\langle f_{j}^{2}, f_{i}^{2}\right\rangle$. Thus, $a^{j} \in \operatorname{Ker} T_{1}^{*}$ and $b^{j} \in \operatorname{Ker} T_{2}^{*}$. But $\operatorname{Ker} T_{1}^{*}=\left(\operatorname{Ran} T_{1}\right)^{\perp} \subset\left(\operatorname{Ran} T_{2}\right)^{\perp}=\operatorname{Ker} T_{2}^{*}$. Therefore $a^{j} \in \operatorname{Ker} T_{2}^{*}$ and then $c^{j}=a^{j}-b^{j} \in \operatorname{Ker} T_{2}^{*}$ which means $T_{2}^{*} c^{j}=0$ or $f_{j}^{2}=J f_{j}^{1}$. Moreover, $T_{2}=T_{1} J^{*}$ and, as we have proved before, $\operatorname{Ker} J=T_{1}^{*}\left(\operatorname{Ran} T_{1} \cap\left(\operatorname{Ran} T_{2}\right)^{\perp}\right)$.

2. The conclusion comes from point 1: the partial isometry will have a zero kernel $(\operatorname{Ker} J=\{0\}$ ) and therefore it is a unitary operator (recall that the range of $J$ should be $H$ ).

This ends the proof of the lemma.

Lemma 2.2. Consider $\mathcal{F}_{1}=\left\{f_{i}^{1}\right\}_{i \in \mathbf{I}}$ and $\mathcal{F}_{2}=\left\{f_{i}^{2}\right\}_{i \in \mathbf{I}}$ two frames in $H$. Let us denote by $T_{1}$ and $T_{2}$ respectively, their analysis operators. Then:

1) Ran $T_{2} \subset R a n T_{1}$ if and only if $\mathcal{F}_{1}$ and $\mathcal{F}_{2}$ are $Q$-partial equivalent for some bounded operator $Q$; furthermore, $\operatorname{Ker} Q=T_{1}^{*}\left(\operatorname{Ran} T_{1} \cap\left(\operatorname{Ran} T_{2}\right)^{\perp}\right)$.

2) $R a n T_{1}=R a n T_{2}$ if and only if $\mathcal{F}_{1}$ and $\mathcal{F}_{2}$ are $Q$-equivalent, for some invertible operator $Q$.

Proof. Let us denote by $S_{1}=T_{1}^{*} T_{1}, S_{2}=T_{2}^{*} T_{2}$ the frame operators.

1. Suppose $\operatorname{Ran} T_{2} \subset \operatorname{Ran} T_{1}$. We have that $\mathcal{F}_{1}$ is $Q$-equivalent with $\mathcal{F}_{1}$ \# $\left(\left(f_{i}^{1}\right)^{\#}=S_{1}^{-1 / 2} f_{i}^{1}\right) ; \mathcal{F}_{1}^{\#}$ is $J$-partial equivalent with $\mathcal{F}_{2}{ }^{\#}$ from Lemma 2.1 , where $J=\left(T_{2}^{\#}\right)^{*} T_{1}^{\#}$ is a partial isometry, and $\mathcal{F}_{2}^{\#}$ is $Q$-equivalent with $\mathcal{F}_{2}$ with $Q=$ $S_{2}^{1 / 2}\left(f_{i}^{2}=S_{2}^{1 / 2}\left(f_{i}^{2}\right)^{\#}\right)$. By composing, we get $\mathcal{F}_{1}$ is $Q$-partial equivalent with $\mathcal{F}_{2}$ via $Q=S_{2}^{1 / 2} J S_{1}^{-1 / 2}$. Furthermore, since $S_{1}$ and $S_{2}$ are invertible, $\operatorname{Ker} Q=$ $S_{1}^{1 / 2} \operatorname{Ker} J=T_{1}^{*}\left(\operatorname{Ran} T_{1} \cap\left(\operatorname{Ran} T_{2}\right)^{\perp}\right)$.

Conversely, if $\mathcal{F}_{1}$ is $Q$-partial equivalent with $\mathcal{F}_{2}$ and $Q$ is the bounded operator relating $\mathcal{F}_{1}$ to $\mathcal{F}_{2}$, then $T_{2}=T_{1} Q^{*}$ and obviously $\operatorname{Ran} T_{2} \subset \operatorname{Ran} T_{1}$. On the other hand, since $T_{1}^{*} T_{1}=S_{1}$ is invertible, $Q=T_{2}^{*} T_{1} S_{1}^{-1}$ and then $\mathcal{F}_{1}^{\#}$ is $J$-partial equivalent with $\mathcal{F}_{2}^{\#}$ with $J=S_{2}^{-1 / 2} Q S_{1}^{1 / 2}$. We have

$$
J J^{*}=S_{2}^{-1 / 2} Q S_{1}^{1 / 2} S_{1}^{1 / 2} Q^{*} S_{2}^{-1 / 2}=S_{2}^{-1 / 2} T_{2}^{*} P_{1} T_{2} S_{2}^{-1 / 2}
$$

where $P_{1}=T_{1} S_{1}^{-1} T_{1}^{*}$ is the orthogonal projection onto $\operatorname{Ran} T_{1}$. But $\operatorname{Ran} T_{2} \subset$ Ran $T_{1}$; hence, $P_{1} T_{2}=T_{2}$. Thus, $J J^{*}=S_{2}^{-1 / 2} T_{2}^{*} T_{2} S_{2}^{-1 / 2}=1$, proving that $J$ is a partial isometry. Now we apply the conclusion of Lemma 2.1 and obtain that $\operatorname{Ker} J=\left(T_{1}^{\#}\right)^{*}\left(\operatorname{Ran} T_{1} \cap\left(\operatorname{Ran} T_{2}\right)^{\perp}\right)$. Substituting this into $\operatorname{Ker} Q=S_{1}^{1 / 2} \operatorname{Ker} J$ we obtain the result.

2. The statement is obtained from 1 ) by observing that $\operatorname{Ker} Q=\{0\}$; since we also know that $\operatorname{Ran} Q=H, Q$ is therefore invertible with bounded inverse. 
We now present the connection between the closeness relation and partial equivalency.

Lemma 2.3. Consider $\mathcal{F}_{1}=\left\{f_{i}^{1}\right\}_{i \in \mathbf{I}}$ and $\mathcal{F}_{2}=\left\{f_{i}^{2}\right\}_{i \in \mathbf{I}}$ two frames in $H$. Let us denote by $T_{1}$ and $T_{2}$, respectively, their analysis operators. Then, $\mathcal{F}_{1}$ is close to $\mathcal{F}_{2}$ (i.e., $\left.c\left(\mathcal{F}_{1}, \mathcal{F}_{2}\right)<\infty\right)$ if and only if $\mathcal{F}_{2}$ is $Q$-partial equivalent with $\mathcal{F}_{1}$ for some bounded operator $Q$ and therefore $\operatorname{Ran} T_{2} \subset \operatorname{Ran} T_{1}$. Moreover, $c\left(\mathcal{F}_{1}, \mathcal{F}_{2}\right)=$ $\|Q-1\|$.

Proof. $\Rightarrow$ Suppose $\mathcal{F}_{1}$ is close to $\mathcal{F}_{2}$. Then $\left\|\sum_{i \in \mathbf{I}} c_{i}\left(f_{i}^{1}-f_{i}^{2}\right)\right\| \leq \lambda\left\|\sum_{i \in \mathbf{I}} c_{i} f_{i}^{2}\right\|$ for $\lambda=c\left(\mathcal{F}_{1}, \mathcal{F}_{2}\right)$. If $c=\left\{c_{i}\right\}_{i \in \mathbf{I}} \in \operatorname{Ker} T_{2}^{*}$, then necessarily $c \in \operatorname{Ker} T_{1}^{*}$. Therefore, $\operatorname{Ker} T_{2}^{*} \subset \operatorname{Ker} T_{1}^{*}$ or $\operatorname{Ran} T_{1}=\left(\operatorname{Ker} T_{1}^{*}\right)^{\perp} \subset\left(\operatorname{Ker} T_{2}^{*}\right)^{\perp}=\operatorname{Ran} T_{2}$. Now, applying Lemma 2.2 we get that $\mathcal{F}_{2}$ is $Q$-partial equivalent with $\mathcal{F}_{1}$. Then, $f_{i}^{1}=Q f_{i}^{2}$ and if we denote $v=\sum_{i \in \mathbf{I}} c_{i} f_{i}^{2}$ we have

$$
\|(Q-1) v\| \leq \lambda\|v\| .
$$

The smallest $\lambda \geq 0$ that satisfies the above inequality for any $v \in H$ is $\|Q-1\|$. Therefore $c\left(\mathcal{F}_{1}, \mathcal{F}_{2}\right)=\|Q-1\|$.

$\Leftarrow$ Suppose $\mathcal{F}_{2}$ is $Q$-partial equivalent with $\mathcal{F}_{1}$. Then, it is easy to check that $c\left(\mathcal{F}_{1}, \mathcal{F}_{2}\right)=\|Q-1\|$ and then $\mathcal{F}_{1}$ is close to $\mathcal{F}_{2}$.

As a consequence of this lemma, we obtain the following result:

Theorem 2.4. Let $\mathcal{F}_{1}$ and $\mathcal{F}_{2}$ be two frames. Then, they are near if and only if they are $Q$-equivalent for some invertible operator $Q$. Moreover, $d^{0}\left(\mathcal{F}_{1}, \mathcal{F}_{2}\right)=$ $\max \left(\|Q-1\|,\left\|1-Q^{-1}\right\|\right)$.

Applying this theorem to the set $\mathcal{T}$ defined in (1.11) we obtain the following corollary:

Corollary 2.5. Consider a frame $\mathcal{G}=\left\{g_{i}\right\}_{i \in \mathbf{I}}$ in $H$ and consider also the set $\mathcal{T}$ defined by (1.11). Then $\mathcal{T}$ is parametrized in the following way:

$$
\mathcal{T}=\left\{\mathcal{F}=\left\{f_{i}\right\}_{i \in \mathbf{I}} \mid f_{i}=\alpha U g_{i}^{\#} \text { where } \alpha>0 \text { and } U \text { is unitary }\right\} .
$$

Proof. Indeed, let $\alpha>0$ and $U$ be unitary. Then, by computing its frame operator one can easily check that $\mathcal{F}=\left\{f_{i}\right\}_{i \in \mathbf{I}}, f_{i}=\alpha U g_{i}^{\#}$ is a tight frame with bound $\alpha^{2}$.

Conversely, suppose $\mathcal{F}=\left\{f_{i}\right\}_{i \in \mathbf{I}} \in \mathcal{T}$. Then, from Theorem 2.4 we obtain $f_{i}=Q g_{i}^{\#}$ for some invertible $Q$. We compute its frame operator:

$$
S^{\mathcal{F}}=\sum_{i \in \mathbf{I}}\left\langle\cdot, f_{i}\right\rangle f_{i}=Q\left(\sum_{i \in \mathbf{I}}\left\langle\cdot, g_{i}^{\#}\right\rangle g_{i}^{\#}\right) Q^{*}=Q Q^{*}
$$

Therefore, $Q Q^{*}=A \cdot 1$ which means that $\frac{1}{\sqrt{A}} Q$ is unitary. Thus $Q=\sqrt{A} U$ for some unitary $U$.

The following result makes a connection between the extension of the Paley and Wiener theorem given by Christensen in [Chr95] and the relations introduced so far.

Theorem 2.6. Let $\mathcal{F}=\left\{f_{i}\right\}_{i \in \mathbf{I}}$ be a frame in $H$ and $\mathcal{G}=\left\{g_{i}\right\}_{i \in \mathbf{I}}$ be a set of vectors in $H$. Suppose there exists $\lambda \in[0,1)$ such that

$$
\left\|\sum_{i \in \mathbf{I}} c_{i}\left(g_{i}-f_{i}\right)\right\| \leq \lambda\left\|\sum_{i \in \mathbf{I}} c_{i} f_{i}\right\|
$$


for any $n \in \mathbf{N}$ and $c_{1}, c_{2}, \ldots$ in $\mathbf{C}$. Then $\mathcal{G}$ is a frame in $H$ and

1) $\mathcal{G}$ is Q-equivalent with $\mathcal{F}$;

2) if $T^{f}$ and $T^{g}$ are the analysis operators associated respectively to $\mathcal{F}$ and $\mathcal{G}$, then $\operatorname{Ran} T^{f}=\operatorname{Ran} T^{g}$;

3) $c(\mathcal{G}, \mathcal{F}) \leq \lambda<1$ and $d^{0}(\mathcal{G}, \mathcal{F})<\infty$.

Proof. The conclusion that $\mathcal{G}$ is a frame follows from a stability result proved by Christensen in [Chr95]. As we have checked before, from $c(\mathcal{G}, \mathcal{F})<1$ we get $c(\mathcal{F}, \mathcal{G}) \leq \frac{\lambda}{1-\lambda}<\infty$. Therefore, $\mathcal{F}$ and $\mathcal{G}$ are near and we can apply Theorem 2.4 and complete the proof.

Theorem 2.4 allows us to partition the set of all frames on $H$, denoted $\mathcal{F}(H)$, into equivalent classes, as follows:

$$
\mathcal{F}(H)=\bigcup_{\alpha \in A} \mathcal{E}_{\alpha}
$$

where $\mathcal{E}_{\alpha} \subset \mathcal{F}(H)$ is a set of frames such that any $\mathcal{F}, \mathcal{G} \in \mathcal{E}_{\alpha}, \mathcal{F}$ is $Q$-equivalent with $\mathcal{G}$ or, equivalent, $\mathcal{F}$ is near to $\mathcal{G}$. Therefore, for each index $\alpha \in A$, the function $d^{0}: \mathcal{E}_{\alpha} \times \mathcal{E}_{\alpha} \rightarrow \mathbf{R}_{+}$is well-defined and finite. We want to prove now that the function

$$
d: \mathcal{E}_{\alpha} \times \mathcal{E}_{\alpha} \rightarrow \mathbf{R}_{+}, d(\mathcal{F}, \mathcal{G})=\log \left(1+d^{0}(\mathcal{F}, \mathcal{G})\right)
$$

is a distance on each class $\mathcal{E}_{\alpha}$.

Theorem 2.7. The function $d$ defined above is a distance on $\mathcal{E}_{\alpha}$. Moreover, for any $\mathcal{F} \in \mathcal{E}_{\alpha}$ and $\mathcal{G} \in \mathcal{F}(H)$, if $d(\mathcal{F}, \mathcal{G})<\infty$, then $\mathcal{G} \in \mathcal{E}_{\alpha}$.

Proof. The second part of the statement is immediate: if $d(\mathcal{F}, \mathcal{G})$ is finite so is $d^{0}(\mathcal{F}, \mathcal{G})$; hence, $\mathcal{F}$ is close to $\mathcal{G}$ and therefore they belong to the same class. To prove that $d$ is a distance we need to check only the triangle inequality. Let $\mathcal{F}, \mathcal{G}, \mathcal{H} \in \mathcal{E}_{\alpha}$. Then, there exist $Q$ and $R$ invertible bounded operators on $H$ such that $g_{i}=Q f_{i}, h_{i}=R g_{i}$ and therefore $h_{i}=R Q f_{i}$. We have

$$
\begin{gathered}
d(\mathcal{F}, \mathcal{G})=\log \left(1+\max \left(\|Q-1\|,\left\|Q^{-1}-1\right\|\right)\right), \\
d(\mathcal{G}, \mathcal{H})=\log \left(1+\max \left(\|R-1\|,\left\|R^{-1}-1\right\|\right)\right), \\
d(\mathcal{F}, \mathcal{H})=\log \left(1+\max \left(\|R Q-1\|,\left\|Q^{-1} R^{-1}-1\right\|\right)\right),
\end{gathered}
$$

and

$$
\begin{aligned}
\|R Q-1\| & =\|(R-1)(Q-1)+R+Q-2\| \\
& \leq\|R-1\| \cdot\|Q-1\|+\|R-1\|+\|Q-1\| \\
& =(\|R-1\|+1)(\|Q-1\|+1)-1 .
\end{aligned}
$$

Hence,

$$
\log (\|R Q-1\|+1) \leq \log (\|R-1\|+1)+\log (\|Q-1\|+1) .
$$

Similarly for $\left\|Q^{-1} R^{-1}-1\right\|$ and therefore $d(\mathcal{F}, \mathcal{H}) \leq d(\mathcal{F}, \mathcal{G})+d(\mathcal{G}, \mathcal{H})$.

The next step is to relate the partition (1.8) with the set of infinite dimensional closed subspaces of $l^{2}(\mathbf{I})$. We suppose $H$ is infinite dimensional and $\mathbf{I}$ is countable. Otherwise, the following result still holds providing we replace "infinite dimensional closed subspaces" by "subspaces of dimension equal to the dimension of $H$ ". 
Let us denote by $\mathcal{S}\left(l^{2}(\mathbf{I})\right)$ the set of all infinite dimensional closed subspaces of $l^{2}(\mathbf{I})$. Then Lemma 2.2 and Theorem 2.4 assert that $\mathcal{F}(H)$ is mapped into $\mathcal{S}\left(l^{2}(\mathbf{I})\right)$ by

$$
i: \mathcal{F}(H) \rightarrow \mathcal{S}\left(l^{2}(\mathbf{I})\right), i\left(\mathcal{E}_{\alpha}\right)=\operatorname{Ran} T
$$

where $T$ is the analysis operator associated to any frame $\mathcal{F} \in \mathcal{E}_{\alpha}$. The natural question that can be asked is whether $i$ is surjective, i.e., if for any closed infinite dimensional subspace of $l^{2}(\mathbf{I})$ we can find a corresponding frame in $\mathcal{F}(H)$. The answer is yes as the following theorem proves (see Christensen [Chr93], Aldroubi [Ald94] or Holub [Hol94] for this type of argument).

Theorem 2.8. For any infinite dimensional closed subspace $E$ of $l^{2}(\mathbf{I})$ there exists a frame $\mathcal{F} \in \mathcal{F}(H)$ (and therefore a class $\mathcal{E}_{\alpha}$ ) such that $i(\mathcal{F})=E$ (in other words, $\operatorname{Ran} T=E$ with $T$ the analysis operator associated to $\mathcal{F})$. Therefore, $i$, considered from the set of classes $\mathcal{E}_{\alpha}$ into $\mathcal{S}\left(l^{2}(\mathbf{I})\right)$, is a bijective mapping.

Proof. Let $E \subset l^{2}(\mathbf{I})$ be an infinite dimensional closed subspace. Choose an orthonormal basis $\left\{d_{i}\right\}_{i \in \mathbf{I}}$ in $E$ and a basis $\left\{e_{i}\right\}_{i \in \mathbf{I}}$ in $H$ (recall $H$ is infinite dimensional and $\mathbf{I}$ countable). Let $p_{i}: l^{2}(\mathbf{I}) \rightarrow \mathbf{C}$ be the canonical projection, $p_{i}(c)=c_{i}$, where $c=\left\{c_{j}\right\}_{j \in \mathbf{I}}$, let $i \in \mathbf{I}$ and $P: l^{2}(\mathbf{I}) \rightarrow \mathbf{C}$ be the canonical projection onto $E$. Let us denote by $\left\{\delta_{i}\right\}_{i \in \mathbf{I}}$ the canonical basis in $l^{2}(\mathbf{I})$, i.e., $\delta_{i}=\left\{\delta_{i j}\right\}_{j \in \mathbf{I}}$. Then, it is known (see [Hol94]) that $\left\{P \delta_{i}\right\}_{i \in \mathbf{I}}$ is a tight frame with bound 1 in $E$ (and any tight frame indexed by $I$ with bound 1 in $E$ is of this form, i.e., the orthogonal projection of some orthonormal basis of $\left.l^{2}(\mathbf{I})\right)$ since

$$
\sum_{i \in \mathbf{I}}\left\langle c, P \delta_{i}\right\rangle P \delta_{i}=P \sum_{i \in \mathbf{I}}\left\langle P c, \delta_{i}\right\rangle \delta_{i}=P c=c, \forall c \in E .
$$

We define a tight frame with bound 1 in $H$ in the following way:

$$
f_{i}=\sum_{j \in \mathbf{I}}\left\langle P \delta_{i}, d_{j}\right\rangle e_{j}=\sum_{j \in \mathbf{I}}\left\langle\delta_{i}, d_{j}\right\rangle e_{j}=\sum_{j_{i}} p_{i}\left(d_{j}\right) e_{j} .
$$

It is easy to prove that $f_{i}$ 's are well defined, since $\left\|f_{i}\right\|^{2}=\sum_{j \in \mathbf{I}}\left|\left\langle P \delta_{i}, d_{j}\right\rangle\right|^{2}$ $=\left\|P \delta_{i}\right\|^{2}<\infty$. Let $T$ be the analysis operator associated to $\left\{f_{i}\right\}_{i \in \mathbf{I}}$ and $x \in H$ be arbitrary. Then

$$
\left\langle x, f_{i}\right\rangle=\sum_{j \in \mathbf{I}} p_{i}\left(d_{j}\right)\left\langle x, e_{j}\right\rangle=p_{i}\left(\sum_{j \in \mathbf{I}}\left\langle x, e_{j}\right\rangle d_{j}\right), \forall i \in \mathbf{I} .
$$

Thus, $T(x)=\left\{\left\langle x, f_{i}\right\rangle\right\}_{i \in \mathbf{I}}=\sum_{j \in \mathbf{I}}\left\langle x, e_{j}\right\rangle d_{j}$ and obvious $\operatorname{Ran} T=E$. It is simple to check that $T f_{i}=P \delta_{i}$ and therefore, $\left\{f_{i}\right\}_{i \in \mathbf{I}}$ is a tight frame with bound 1 .

\section{Minimal Distances Between A GIVEn FRAME AND A TIGHT FRAME}

We are concerned here with the closeness and distance functions $d^{1}, d^{2}$ and $\left.d\right|_{\mathcal{T}}$ introduced earlier. In fact, we would like to characterize the minima of these functions. Here is the main result:

Theorem 3.1. Consider $\mathcal{G}=\left\{g_{i}\right\}_{i \in \mathbf{I}}$ a frame in $H$ with optimal frame bounds $A, B$ and consider the sets $\mathcal{T}^{1}, \mathcal{T}^{2}$ and $\mathcal{T}$ introduced in (1.9), (1.10) and (1.11). Let us denote by $\theta=\frac{\sqrt{B}-\sqrt{A}}{\sqrt{B}+\sqrt{A}}$ and $\rho=\frac{1}{4}(\log B-\log A)$. Then the following conclusions hold: 
1. The values of the minima of $d^{1}, d^{2}$ and $\left.d\right|_{\mathcal{T}}$ are given by

$$
\min d^{1}=\min d^{2}=\left.\theta \min d\right|_{\mathcal{T}}=\rho .
$$

2. These values are achieved by the following scalings of the associated tight frames of $\mathcal{G}$ :

$$
\begin{gathered}
\mathcal{F}^{1}=\left\{f_{i}^{1}\right\}_{i \in \mathbf{I}}, f_{i}^{1}=\frac{\sqrt{A}+\sqrt{B}}{2} g_{i}^{\#}, \\
\mathcal{F}^{2}=\left\{f_{i}^{2}\right\}_{i \in \mathbf{I}}, f_{i}^{2}=\frac{2 \sqrt{A B}}{\sqrt{A}+\sqrt{B}} g_{i}^{\#}, \\
\mathcal{F}^{0}=\left\{f_{i}^{0}\right\}_{i \in \mathbf{I}}, f_{i}^{0}=\sqrt[4]{A B} g_{i}^{\#} .
\end{gathered}
$$

Hence, $d^{1}\left(\mathcal{F}^{1}\right)=d^{2}\left(\mathcal{F}^{2}\right)=\theta$ and $d\left(\mathcal{F}^{0}\right)=\rho$.

3. Any tight frame that achieves the minimum of one of the three functions $d^{1}$, $d^{2}$ or $d$ is unitarily equivalent with the corresponding solution (3.1), (3.2) or (3.3) in the following way:

$$
\begin{gathered}
\left(d^{1}\right)^{-1}(\theta)=\left\{\mathcal{K}=\left\{k_{i}\right\}_{i \in \mathbf{I}} \mid k_{i}=U f_{i}^{1},\right. \\
\left.U \text { unitary and }\left\|U-\frac{2}{\sqrt{A}+\sqrt{B}} S^{1 / 2}\right\|=\theta\right\}, \\
\left(d^{2}\right)^{-1}(\theta)=\left\{\mathcal{K}=\left\{k_{i}\right\}_{i \in \mathbf{I}} \mid k_{i}=U f_{i}^{2},\right. \\
\left.U \text { unitary and }\left\|U-\frac{2 \sqrt{A B}}{\sqrt{A}+\sqrt{B}} S^{-1 / 2}\right\|=\theta\right\}, \\
d^{-1}(\rho)=\left\{\mathcal{K}=\left\{k_{i}\right\}_{i \in \mathbf{I}} \mid k_{i}=U f_{i}^{0},\right. \\
\left.U \text { unitary and }\left\|U-\sqrt[4]{A B} S^{-1 / 2}\right\|=\left\|U-\frac{1}{\sqrt[4]{A B}} S^{1 / 2}\right\|=\rho\right\}
\end{gathered}
$$

where $S$ is the frame operator associated to $\mathcal{G}$. Moreover, any unitary operator that parametrizes $\left(d^{1}\right)^{-1}(\theta),\left(d^{2}\right)^{-1}(\theta)$ or $d^{-1}(\rho)$ as above, has the value 1 in its spectrum.

Proof. If $\mathcal{G}$ is a tight frame, then $\mathcal{F}^{1}=\mathcal{F}^{2}=\mathcal{F}^{0}=\mathcal{G}$ and $\theta=\rho=0$ and the problem is solved. Therefore, we may suppose that $A<B$.

We prove this in the following way: In the first step we check that $d^{1}\left(\mathcal{F}^{1}\right)=$ $d^{2}\left(\mathcal{F}^{2}\right)=\theta$ and $d\left(\mathcal{F}^{0}\right)=\rho$. Then, since $\theta<1$, it follows that the infimum of $d^{1}$ and $d^{2}$ are less than 1. Now, using Corollary 2.5 and Theorem 2.4 we can reduce our problem to an infimum of an operator norm. In the third step we will prove two lemmas, one to be applied to $d^{1}$ and $d^{2}$, and the other to $d$, and this will end the proof.

i) Let us check that (3.1), (3.2), (3.3) achieve the desired values for $d^{1}, d^{2}$ and $d$, respectively. For $f_{i}^{1}=Q g_{i}$ with $Q=\frac{\sqrt{A}+\sqrt{B}}{2} S^{-1 / 2}$ we have $d^{1}\left(\mathcal{F}^{1}\right)=c\left(\mathcal{G}, \mathcal{F}^{1}\right)=$ $\left\|1-Q^{-1}\right\|$. Now, $\sqrt{A} \leq S^{1 / 2} \leq \sqrt{B}$ where the inequalities cannot be improved. Therefore,

$$
-\frac{\sqrt{B}-\sqrt{A}}{\sqrt{B}+\sqrt{A}} \leq 1-Q^{-1} \leq \frac{\sqrt{B}-\sqrt{A}}{\sqrt{B}+\sqrt{A}}
$$


which means that $\left\|1-Q^{-1}\right\|=\theta$. Similarly, for $f_{i}^{2}=L g_{i}$ with $L=\frac{2 \sqrt{A B}}{\sqrt{A}+\sqrt{B}} S^{-1 / 2}$ we have $d^{2}\left(\mathcal{F}^{2}\right)=c\left(\mathcal{F}^{2}, \mathcal{G}\right)=\|L-1\|$ and a similar calculus shows that $d^{2}\left(\mathcal{F}^{2}\right)=\theta$.

For $\mathcal{F}^{0}$ we have $f_{i}^{0}=R g_{i}$ with $R=\sqrt[4]{A B} S^{-1 / 2}$ and therefore

$$
d\left(\mathcal{F}^{0}\right)=\log \left(1+\max \left(\|R-1\|,\left\|1-R^{-1}\right\|\right)\right) .
$$

Now, an easy calculation shows that

$$
\|R-1\|=\left\|1-R^{-1}\right\|=\max \left(\sqrt[4]{\frac{B}{A}}-1,1-\sqrt[4]{\frac{A}{B}}\right)=\sqrt[4]{\frac{B}{A}}-1 .
$$

Therefore, $d\left(\mathcal{F}^{0}\right)=\log \sqrt[4]{\frac{B}{A}}=\rho$.

ii) Since we are looking for the infimum of the functions $d^{1}, d^{2}$ and since $\theta<1$ we may then restrict our attention to only the tight frames $\mathcal{F} \in \mathcal{T}^{1}$ (or to $\mathcal{T}^{2}$ ) such that $d^{1}(\mathcal{F})<1$ (respectively, $\left.d^{2}(\mathcal{F})<1\right)$. But this implies also that $d^{2}(\mathcal{F})<\infty$ (respectively, $\left.d^{1}(\mathcal{F})<\infty\right)$. Therefore, we may restrict our attention only to tight frames in $\mathcal{T}^{1} \cap \mathcal{T}^{2}=\mathcal{T}$.

Corollary 2.5 tells us that these frames must have the form $\mathcal{F}=\left\{f_{i}\right\}_{i \in \mathbf{I}}$ and $f_{i}=\sqrt{C} U g_{i}^{\#}=\sqrt{C} U S^{-1 / 2} g_{i}$ for some $C>0$ and $U$ unitary. Hence

$$
\begin{gathered}
d^{1}(\mathcal{F})=\left\|1-\frac{1}{\sqrt{C}} S^{1 / 2} U^{-1}\right\|=\left\|\frac{1}{\sqrt{C}} S^{1 / 2}-U\right\|, \\
d^{2}(\mathcal{F})=\left\|\sqrt{C} U S^{-1 / 2}-1\right\|=\left\|\sqrt{C} S^{1 / 2}-U\right\|, \\
d^{0}(\mathcal{F})=\max \left(\left\|\frac{1}{\sqrt{C}} S^{1 / 2}-U\right\|,\left\|\sqrt{C} S^{-1 / 2}-U\right\|\right) .
\end{gathered}
$$

To minimize $d$ is equivalent to minimizing $d^{0}$; since $d^{0}$ has a simpler expression, we prefer to work with $d^{0}$ from now on.

Thus, our problem is reduced to find minima of the operator norms (3.7), (3.8), (3.9) subject to $C>0$ and $U$ unitary.

iii) The next step is to solve these norm problems. For $d^{1}$ and $d^{2}$ we apply the following lemma to be proved later:

Lemma 3.2. Consider $R$ a selfadjoint operator on $H$ with $a=\left\|R^{-1}\right\|^{-1}$ and $b=$ $\|R\|$. Then, the solution of the following inf-problem

$$
\mu=\inf _{\substack{\alpha>0 \\ u \text { unitary }}}\|\alpha R-U\|
$$

is given by $\mu=\frac{b-a}{b+a}$ and $\alpha=\frac{2}{a+b}$. This infimum is achieved by the identity operator; any other unitary $U$ that achieves the infimum must have 1 in its spectrum.

If we apply this lemma with $R=S^{1 / 2}, \alpha=\frac{1}{\sqrt{C}}$ and $a=\sqrt{A}, b=\sqrt{B}$, then we get $\mu=\frac{\sqrt{B}-\sqrt{A}}{\sqrt{B}+\sqrt{A}} \equiv \theta$ and $\alpha=\frac{2}{\sqrt{A}+\sqrt{B}}$, hence the parametrization (3.4) of the solutions. This proves (3.7. For (3.8) we apply the lemma with $R=S^{-1 / 2}, \alpha=\sqrt{C}$ and $a=\frac{1}{\sqrt{B}}, b=\frac{1}{\sqrt{A}}$. We get $\mu=\theta$ and $\alpha=\frac{2 \sqrt{A B}}{\sqrt{A}+\sqrt{B}}$, hence the parametrization (3.5) of the solutions.

For $d$ we need a similar lemma, but this time for another optimization problem: 
Lemma 3.3. Consider $R$ a bounded invertible selfadjoint operator on $H$ with $a=$ $\left\|R^{-1}\right\|^{-1}$ and $b=\|R\|$. Then, the solution of the following optimization problem:

$$
\mu=\inf _{\substack{\alpha>0 \\ \text { unitary }}} \max \left(\|\alpha R-U\|,\left\|\frac{1}{\alpha} R^{-1}-U\right\|\right)
$$

is given by $\mu=\sqrt{\frac{b}{a}}-1, \alpha=\frac{1}{\sqrt{a b}}$ and $U$ in the set

$$
\left\{U: H \rightarrow H \mid U \text { unitary and }\left\|\frac{1}{\sqrt{a b}} R-U\right\|=\left\|\sqrt{a b} R^{-1}-U\right\|=\sqrt{\frac{b}{a}}-1\right\} .
$$

Moreover, the set (3.15) contains the identity and therefore, is not empty and the spectrum of any $U$ contains 1 .

The solution for $d^{0}$ is now straightforward: we apply this lemma to (3.9) with $R=S^{1 / 2}, \alpha=\frac{1}{\sqrt{C}}$ and $a=\sqrt{A}, b=\sqrt{B}$. We get $\mu=\min d^{0}=\sqrt[4]{\frac{B}{A}}-1$ and $\alpha=\frac{1}{\sqrt[4]{A B}}$, hence the parametrization (3.6) of the solution and the proof of theorem is complete.

It still remains to prove the two lemmas.

Proof of Lemma 3.2. Let $\delta=\alpha-\frac{2}{a+b}$. We denote by $\sigma(X)$ the spectrum of the operator $X$. Thus, $a, b \in \sigma(R)$. Now, by Weyl's criterion (see for instance, [ReSi80]), there are two sequences of normed vectors in $H,\left(v_{n}\right)_{n \in \mathbf{N}}$ and $\left(w_{n}\right)_{n \in \mathbf{N}}$ such that $\left\|v_{n}\right\|=\left\|w_{n}\right\|=1$ and $\lim _{n}\left\|(R-a) v_{n}\right\|=0, \lim _{n}\left\|(R-b) w_{n}\right\|=0$.

Consider $\delta>0$. Let $\varepsilon=\frac{\delta}{2} b$. Then there exists an index $N$ such that for any $n>N,\left\|R w_{n}-b w_{n}\right\| \leq \frac{\varepsilon}{\alpha}$. We get $\left\|\alpha R w_{n}\right\| \geq \alpha b-\varepsilon>1$ and

$\left\|(\alpha R-U) w_{n}\right\| \geq\left|\left\|\alpha R w_{n}\right\|-\left\|U w_{n}\right\|\right|=\left\|\alpha R w_{n}\right\|-1 \geq \alpha b-\varepsilon-1=\frac{b-a}{b+a}+\varepsilon$.

Therefore,

$$
\|\alpha R-U\| \geq \frac{b-a}{b+a}+\varepsilon>\frac{b-a}{b+a}=\mu .
$$

Consider now $\delta<0$. Let $\varepsilon=-\frac{\delta}{2} a>0$. Then, there exists an $N$ such that for any $n>N,\left\|R v_{n}-a v_{n}\right\| \leq \frac{\varepsilon}{\alpha}$. We get $\left\|\alpha R v_{n}\right\| \leq \alpha a+\varepsilon<1$ and

$\left\|(\alpha R-U) v_{n}\right\| \geq\left|\left\|\alpha R v_{n}\right\|-\left\|U v_{n}\right\|\right|=1-\left\|\alpha R v_{n}\right\| \geq 1-\alpha a-\varepsilon=\frac{b-a}{b+a}+\varepsilon$.

Therefore,

$$
\|\alpha R-U\| \geq \frac{b-a}{b+a}+\varepsilon>\frac{b-a}{b+a}=\mu .
$$

From (3.13) and (3.14) we observe that the infimum of $\|\alpha R-U\|$ has the value $\frac{b-a}{b+a}$ and may be achieved only if $\delta=0$, i.e., $\alpha=\frac{2}{a+b}$. Thus, the first part of the lemma has been proved.

The set of all unitary $U$ that achieves the infimum is then given by

$$
\left\{U: H \rightarrow H \mid U \text { unitary and }\left\|\frac{2}{a+b} R-U\right\|=\frac{b-a}{b+a}\right\} .
$$

We still have to prove that the set (3.15) contains the identity and 1 is in spectrum of any unitary operator from this set. 
From $a \leq R \leq b$ we get $-\frac{b-a}{b+a} \leq \frac{2}{a+b} R-1 \leq \frac{b-a}{b+a}$. Therefore, $\left\|\frac{2}{a+b} R-1\right\| \leq \frac{b-a}{b+a}$. But, us we have proved, $\frac{b-a}{b+a}$ is the minimum that can be achieved. Therefore, $\left\|\frac{2}{a+b} R-1\right\|=\frac{b-a}{b+a}=\mu$ and thus, 1 is in the set (3.15).

Now recall the sequence $\left(v_{n}\right)_{n}$ and the inequality (3.13) which is realized on $\left(v_{n}\right)_{n}$. For $U$ in the set $(3.15)$ we have $\left\|\left(\frac{2}{a+b} R-U\right) v_{n}\right\| \rightarrow \mu$. But

$$
\left\|\left(\frac{2}{a+b} R-U\right) v_{n}\right\|^{2}=\frac{4}{(a+b)^{2}}\left\langle v_{n}, R^{2} v_{n}\right\rangle-\frac{2}{a+b}\left\langle v_{n},\left(R U+U^{*} R\right) v_{n}\right\rangle+1 .
$$

From $(R-a) v_{n} \rightarrow 0$ we get $\left\langle v_{n}, R^{2} v_{n}\right\rangle \rightarrow a^{2}$. Therefore,

$$
\lim _{n}\left\langle v_{n},\left(R U+U^{*} R\right) v_{n}\right\rangle=\frac{a+b}{2}\left(\frac{4 a^{2}}{(a+b)^{2}}+1-\sigma^{2}\right)=2 a .
$$

Now:

$$
R U+U^{*} R=(R-a) U+U^{*}(R-a)+a\left(U+U^{*}\right)
$$

and the previous limit gives $\lim _{n}\left\langle v_{n},\left(U+U^{*}\right) v_{n}\right\rangle=2$.

Therefore,

$$
\left\|(U-1) v_{n}\right\|^{2}=\left\langle v_{n},\left(2-\left(U+U^{*}\right)\right) v_{n}\right\rangle \rightarrow 0
$$

or $\lim _{n}\left\|(U-1) v_{n}\right\|=0$ which proves $1 \in \sigma(U)$.

Proof of Lemma 3.3. First, let us solve the following scalar problem:

$$
\bar{\mu}=\inf _{\alpha>0} \max \left(\max _{a \leq x \leq b}|\alpha x-1|, \max _{a \leq x \leq b}\left|\frac{1}{\alpha x}-1\right|\right) .
$$

Because of monotonicity,

$$
\begin{aligned}
& \max _{a \leq x \leq b}|\alpha x-1|=\max (|\alpha a-1|,|\alpha b-1|), \\
& \max _{a \leq x \leq b}\left|\frac{1}{\alpha x}-1\right|=\max \left(\left|\frac{1}{\alpha a}-1\right|,\left|\frac{1}{\alpha b}-1\right|\right) .
\end{aligned}
$$

Therefore, $\bar{\mu}=\inf _{\alpha>0} f(\alpha)$ where

$$
f(\alpha)=\max \left(|\alpha a-1|,|\alpha b-1|,\left|\frac{1}{\alpha a}-1\right|,\left|\frac{1}{\alpha b}-1\right|\right) .
$$

It is now simpe to check that the infimum may be achieved only when at least two moduli are equal. This condition is fulfilled at the following points:

$$
\alpha_{1}=\frac{2}{a+b} ; \alpha_{2}=\frac{1}{a} ; \alpha_{3}=\frac{1}{a} \pm \frac{1}{a} \sqrt{1-\frac{a}{b}} ; \alpha_{4}=\frac{1}{\sqrt{a b}} ; \alpha_{5}=\frac{1}{b} ; \alpha_{6}=\frac{a+b}{2 a b} \text {. }
$$

We evaluate $f(\alpha)$ at these points and we get

$$
\begin{gathered}
f\left(\alpha_{1}\right)=\frac{b-a}{2 a} ; f\left(\alpha_{2}\right)=\frac{b-a}{a} ; f\left(\alpha_{3}\right)=\frac{\sqrt{b-a}}{a}(\sqrt{b}-\sqrt{b-a}), \\
f\left(\alpha_{4}\right)=\sqrt{\frac{b}{a}}-1 ; f\left(\alpha_{5}\right)=\frac{b-a}{a} ; f\left(\alpha_{6}\right)=\frac{b-a}{2 a} .
\end{gathered}
$$

It is obvious now that $f\left(\alpha_{4}\right) \leq f\left(\alpha_{1}\right)=f\left(\alpha_{6}\right) \leq f\left(\alpha_{2}\right)=f\left(\alpha_{5}\right) \leq f\left(\alpha_{3}\right)$ and therefore, $\bar{\mu}=f\left(\alpha_{4}\right)=\sqrt{\frac{b}{a}}-1$ and $\alpha_{\text {optim }}=\alpha_{4}=\frac{1}{\sqrt{a b}}$. Observe also that for $\alpha=\alpha_{4}$ we have

$$
\max _{a \leq x \leq b}\left|\alpha_{4} x-1\right|=\max _{a \leq x \leq b}\left|\frac{1}{\alpha_{4} x}-1\right|
$$


Let us now return to the norm problem (3.11). Our claim is that the infimum is achieved for $\alpha=\frac{1}{\sqrt{a b}}=\alpha_{4}$ and $U=1$ (the identity) and the value of the infimum is $\mu=\sqrt{\frac{b}{a}}-1=\bar{\mu}$. The solution of the scalar problem (3.16) proves also that the set (3.12) contains the identity.

We are now going to prove that $\mu=\bar{\mu}$ is the optimum and $\alpha=\alpha_{4}$. As in the previous lemma, consider $\left(v_{n}\right)_{n \geq 1}$ and $\left(w_{n}\right)_{n \geq 1}$ two sequences of normed vectors in $H\left(\left\|v_{n}\right\|=\left\|w_{n}\right\|=1\right)$ such that $\lim _{n}\left\|(R-a) v_{n}\right\|=0, \lim _{n}\left\|(R-b) w_{n}\right\|=0$. It is simple to check that $\lim _{n}\left\|\left(R^{-1}-\frac{1}{a}\right) v_{n}\right\|=0$ and $\lim _{n}\left\|\left(R^{-1}-\frac{1}{b}\right) w_{n}\right\|=0$ hold also. Now, consider some $\alpha>0, \alpha \neq \alpha_{4}=\frac{1}{\sqrt{a b}}$. Then, as the scalar problem proved, we have

$$
\text { either } \max _{a \leq x \leq b}|\alpha x-1|>\bar{\mu} \text { or } \max _{a \leq x \leq b}\left|\frac{1}{\alpha x}-1\right|>\bar{\mu} \text {. }
$$

Suppose the first inequality holds. Now, either $|\alpha a-1|>\bar{\mu}$ or $|\alpha b-1|>\bar{\mu}$. In the former case we use the sequence $\left(v_{n}\right)_{n}$ as follows: Let $\varepsilon=\frac{1}{2}(|\alpha a-1|-\bar{\mu})>0$ and let $N_{\varepsilon}$ be such that $\left\|(R-a) v_{n}\right\| \leq \frac{\varepsilon}{\alpha}$ for any $n \geq N_{\varepsilon}$. Then

$$
\begin{gathered}
\left\|(\alpha R-U) v_{n}\right\| \geq\left|\left\|\alpha R v_{n}\right\|-\left\|U v_{n}\right\|\right|=\left|\alpha\left\|a v_{n}+(R-a) v_{n}\right\|-1\right| \\
\geq|\alpha a-1|-\alpha\left\|(R-a) v_{n}\right\|>\bar{\mu}+\varepsilon
\end{gathered}
$$

which implies $\|\alpha R-U\|>\bar{\mu}+\varepsilon$.

Similarly, in the later case $(|\alpha b-1|>\bar{\mu})$ we take $\varepsilon=\frac{1}{2}(|\alpha b-1|-\bar{\mu})>0$ and $N_{\varepsilon}$ such that $\left\|(R-b) w_{n}\right\| \leq \frac{\varepsilon}{\alpha}$ for any $n \geq N_{\varepsilon}$. Therefore,

$$
\begin{gathered}
\left\|(\alpha R-U) w_{n}\right\| \geq\left|\left\|\alpha R w_{n}\right\|-\left\|U w_{n}\right\|\right|=\left|\alpha\left\|b w_{n}+(R-b) w_{n}\right\|-1\right| \\
\geq|\alpha b-1|-\alpha\left\|(R-b) w_{n}\right\|>\bar{\mu}+\varepsilon .
\end{gathered}
$$

Thus, in both cases we obtain $\|\alpha R-U\|>\bar{\mu}$. If the second inequality in (3.17) holds, a similar argument can be used to prove that, for $\alpha \neq \alpha_{4}$ we have

$$
\left\|\frac{1}{\alpha} R^{-1}-U\right\|>\bar{\mu}
$$

Therefore, the optimum in (3.11) is achieved for $\alpha=\frac{1}{\sqrt{a b}}$ and the value of it is $\mu=\sqrt{\frac{b}{a}}-1$. It is obvious now that the set of unitary operators that achieve the optimum is given by (3.12) and also that the identity operator is in that set. The only problem that still remains to be proved is that all these unitary operators have 1 in their spectra.

The previous argument proves the following conclusion: fix $\delta_{0}>$ small enough and let $U$ be in the set (3.12). Then, for any $0<\delta \leq \delta_{0}$ the following inequality holds:

$$
\bar{\mu} \leq\left\|\left(\delta R+\frac{1}{\sqrt{a b}} R-U\right) w_{n}\right\|
$$

for $n \geq N_{\delta}$ where $N_{\delta}$ is an integer depending on $\delta$. Then, $\bar{\mu} \leq\left\|\left(\delta R+\frac{1}{\sqrt{a b}} R-U\right) w_{n}\right\|$ $<\delta\|R\|+\bar{\mu}$ for $n \geq N_{\delta}$, and it is fairly easy to prove now that $\left\|\left(\frac{1}{\sqrt{a b}} R-U\right) w_{n}\right\| \rightarrow$ $\bar{\mu}$ when $n \rightarrow \infty$. Now, by repeating the argument given in the previous lemma we obtain $\lim _{n}\left\|(U-1) w_{n}\right\|=0$ which proves $1 \in \sigma(U)$ and the lemma is proved. 


\section{Conclusions}

In this paper we introduced and studied a distance between Hilbert frames having the same index set $\mathbf{I}$. This distance partitions the set of frames into equivalency classes characterized (and indexed) by closed subspaces of the space of coefficients $l^{2}(\mathbf{I})$. Thus, two frames are at a finite distance if and only if their analysis operators have the same (closed) range in $l^{2}(\mathbf{I})$ and this happens if and only if there exists a bounded and invertible operator on the Hilbert space that maps one frame set into the other.

Next we determined the closest, respectively nearest, tight frame to a given frame. It turns out that these tight frames are scaled versions of the associated tight frame.

We point out that the entire theory can be carried out on the set of Hilbert frames over different Hilbert spaces, but indexed by the same index set. All the results are similar, the changes being straightforward.

As a final remark we acknowledge that Lemmas 2.1 and 2.2 have also been independently obtained by D. Han and D. R. Larson in a recent paper ([HaLa97]).

\section{REFERENCES}

[Ald94] A.Aldroubi, Portraits of Frames, Proc.Amer.Math.Soc., vol.123, no.6 (1995), 16611668. MR 95g: 46037

[AAG93] S.T.Ali, J.-P.Antoine, J.-P.Gazeau, Continuous Frames in Hilbert Space, Annals of Physics, no.1, 222 (1993), 1-37. MR 94e:81107

[Chr93] O.Christensen, Frame Decomposition in Hilbert Spaces, Ph.D. Thesis (1993) http@tyche.mat.univie.ac.at

[Chr95] O.Christensen, A Paley-Wiener Theorem for Frames, Proc.Amer.Math.Soc. 123 (1995), 2199-2202. MR 95i:46027

[ChHe96] O.Christensen, C.Heil, Perturbations of Banach Frames and Atomic Decompositions, Math.Nach., 185 (1997), 33-47 or http@tyche.mat.univie.ac.at. CMP 97:13

[DuEa42] R.J.Duffin, J.J.Eachus, Some Notes on an Expansion Theorem of Paley and Wiener, Bull.Amer.Math.Soc., 48 (1942), 850-855. MR 97e:424x

[DuSc52] R.J.Duffin, A.C.Schaeffer, A Class of Nonharmonic Fourier Series, Trans.Amer.Math.Soc., 72 (1952), 341-366. MR 13:839a

[HaLa97] D.Han, D.R.Larson, Frames, Bases and Group Representations, preprint 1997

[Hol94] J.R.Holub, Pre-Frame Operators, Besselian Frames, and Near-Riesz Bases in Hilbert Spaces, Proc.Amer.Math.Soc. 122, no.3 (1994), 779-785. MR 95a:46030

[Kato76] T.Kato, Perturbation Theory for Linear Operators, Springer-Verlag (1976). MR 53:11389

[PaWi34] R.E.A.C.Paley, N.Wiener, Fourier Transforms in the Complex Domain, AMS Colloq.Publ., vol.19, AMS, Providence R.I. (1934), reprint 1960. MR 98a:01023

[Ron96] A.Ron, Z.Shen, Frames and stable bases for shift-invariant subspaces of $L_{2}\left(\mathbf{R}^{d}\right)$, CMS-TSR \#94-07, University of Winsconsin - Madison, February 1996 or ftp anonymous@stolp.cs.wisc.edu. MR 96k:42049

[ReSi80] M.Reed, B.Simon, Functional Analysis, vol.1, Academic Press (1980). MR 58:12429a

[You80] R.M.Young, An Introduction to Nonharmonic Fourier Series, Academic Press (1980). MR 81m:42027

Program in Applied and Computational Mathematics, Princeton University, PrinceTON, NEW JERSEY 08544

E-mail address: rvbalan@math.princeton.edu 